\title{
Optimization of cutting parameters on turning process based on surface roughness using response surface methodology.
}

\begin{abstract}
This paper describes effect of cutting parameters on surface roughness for turning of aluminium alloy 7050 using carbide cutting tool with dry cutting condition. The model is developed based on cutting speed, feed rate and depth of cut as the parameters of cutting process. The selection of cutting process was based on the design of experiments Response Surface Methodology (RSM). The objective of this research is finding the optimum cutting parameters based on surface roughness. The relation between cutting parameters and surface roughness were discussed.
\end{abstract}

Keyword: Turning process; Parameters process; Surface roughness; Design of experiment. 